\title{
Miniaturized Circularly Polarized Microstrip RFID Antenna Using Fractal Metamaterial
}

\author{
Guo Liu, Liang Xu, and Zhensen Wu \\ Institute of Radio Wave Propagation, School of Science, Xidian University, Xi'an 710071, China \\ Correspondence should be addressed to Guo Liu; liuguosgg@hotmail.com
}

Received 25 January 2013; Accepted 3 April 2013

Academic Editor: Duixian Liu

Copyright ( 2013 Guo Liu et al. This is an open access article distributed under the Creative Commons Attribution License, which permits unrestricted use, distribution, and reproduction in any medium, provided the original work is properly cited.

\begin{abstract}
A novel miniaturized circularly polarized (CP) microstrip antenna that can handle UHF band (920-925 MHz, corresponding to the assigned band for RFID in China) has been designed, fabricated, and measured in this paper. The miniaturization of antenna is achieved by a special cross-shaped fractal metamaterial structure that is inserted between the patch and ground plane. The measured results show that the antenna possesses an impedance bandwidth of $8.7 \%$ with VSWR $\leqslant 1.5: 1$ and 3-dB axial bandwidth of 3.8\%. Furthermore, the proposed antenna has $10.2 \%$ size reduction compared with traditional patch antenna. The tested results are in good agreement with that of the simulations.
\end{abstract}

\section{Introduction}

The radio-frequency identification (RFID) is a type of noncontact two-way data communications, target identification, and access to relevant data to the automatic identification technology by the radio frequency. Recently, RFID systems become more and more popular in all kinds of fields such as logistics, security, and chain management. As is well known, several frequency bands have been assigned to RFID application; there are $125 \sim 135 \mathrm{KHz}, 13.56 \mathrm{MHz}, 2.45 \mathrm{GHz}, 5.8 \mathrm{GHz}$, and some UHF frequencies such as $902 \sim 928 \mathrm{MHz}$ (USA), 950 956 MHz (Japan), 866-869 MHz (Europe), and 840 $845 \mathrm{MHz}, 920 \sim 925 \mathrm{MHz}$ (China) [1]. Microstrip antennas are attractive in RFID systems because of their low cost and low profile and can be integrated with other planer components. However, nowadays, there have more critical requirements for the microstrip antennas as follows: circularly polarized characteristics and smaller miniaturized size. Different techniques have been reported to implement miniaturization of microstrip antennas such as high dielectric constant substrates, shorted probes, and slotted line [2-4]. Those methods are implemented at the expense of the bandwidth, radiation efficiency, or other antenna performance.

In this paper, a different technique is proposed to obtain the miniaturization of microstrip antenna by inserting a special cross-shaped structure between the patch and ground plane. In fact, this structure is a kind of LHM (left-handed metamaterial). The LHM is a kind of medium in which both the permittivity and permeability are simultaneously negative. Many researches have indicated that the LHM has many strange properties, such as the reversed Doppler shift, the reversed Cherenkov radiation, negative refraction, and perfect lens. The term fractal was originally coined by Mandelbrot to describe a family of complex shapes that possess an inherent self-similarity in their geometrical structure [5]. Combining aspects of the modern theory of fractal geometry with antenna design has received a lot of attention, which is known as fractal electrodynamics. A microstrip antenna with fractal multilayer substrates had been reported in [6]. A new type of fractal antenna named tree-like antenna was introduced in 1999 [7]. In this paper, a novel structure using cross-shaped fractal LHM concept is constructed and applied to microstrip antenna. The simulated results have demonstrated that the designed structure has negative permeability. The backward wave property of LHM is employed to compensate for the phase shift resulting from waves propagating in the conventional dielectric medium. In practice, a cross-shaped fractal LHM structure is constructed and applied to implement the miniaturization of microstrip CP RFID antenna. Details of the antenna design and the 


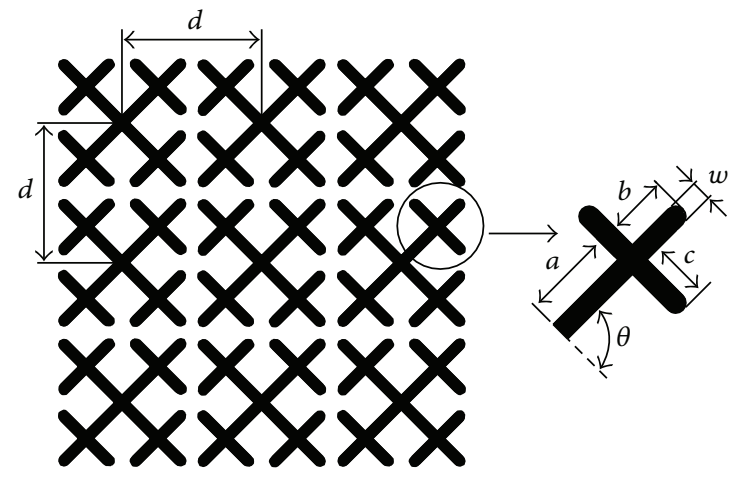

(a)

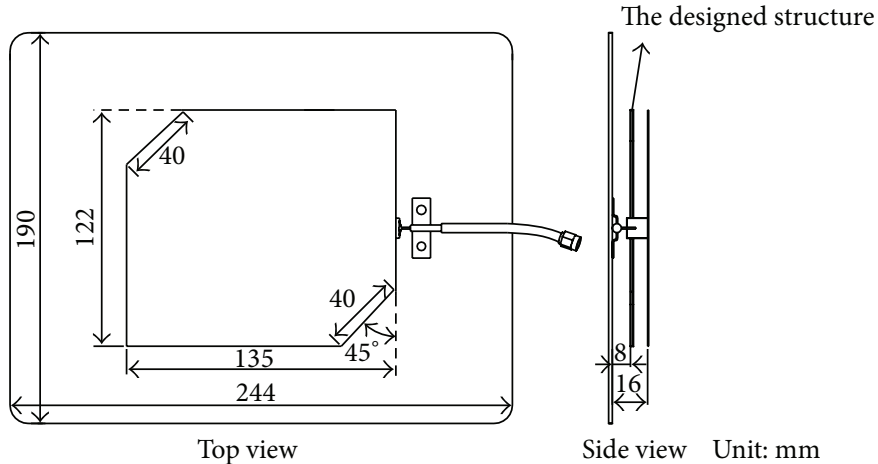

(b)

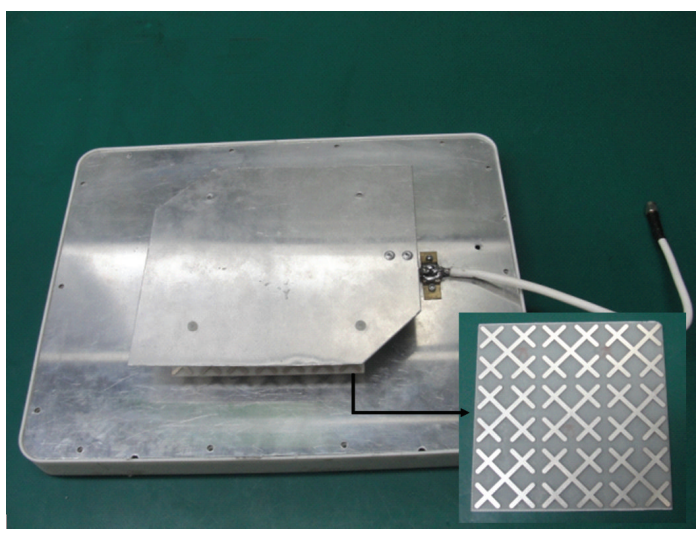

(c)

FIGURE 1: (a) The structure of second-iteration cross-shaped structure. (b) Geometry of the proposed antenna. (c) Photograph of the fabricated antenna.

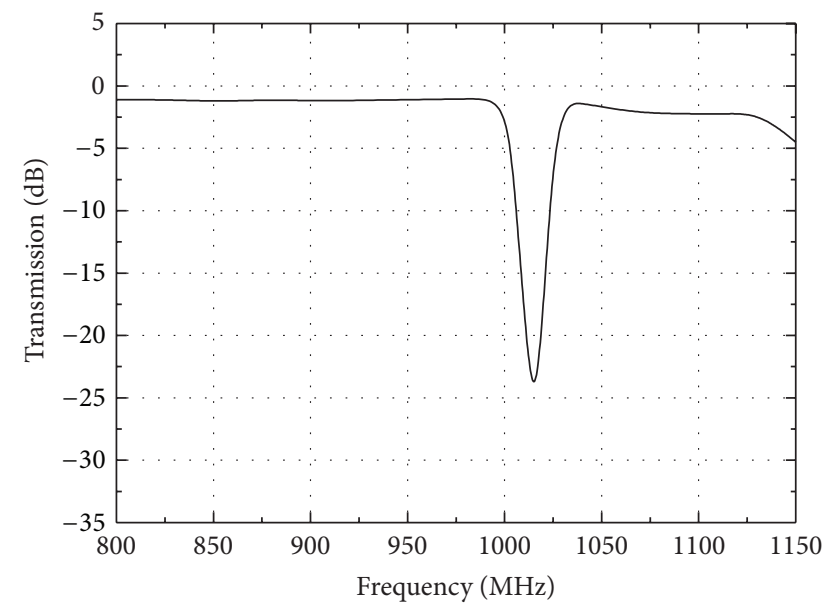

FIGURE 2: The transmission coefficient of the cross-shaped structure.

performance for the antenna with fractal LHM structure are presented and discussed.

\section{Antenna Configurations}

Figure 1(a) shows the geometry of the proposed cross-shaped fractal LHM structure. The designed structure is printed on
RT/Duroid substrate with $1.5 \mathrm{~mm}$ thickness, 2.33 dielectric constant, and 0.001 loss tangent. It is established that the cross-structure in both sides must be aligned strictly. When the electromagnetic wave propagates normally to the surface, magnetic field can cause induced current thereby acted as inductance. Meanwhile, the gap between the cross on both sides can produce capacitance and thus lead to an LC resonance which is related to the parameters $a, b, c$, $d$, and $\theta$. The proposed structure has been designed and optimized using the software CST microwave studio. The final optimized dimensions of the cross-shaped structure are $a=8.62 \mathrm{~mm}, b=5.93 \mathrm{~mm}, c=5.93 \mathrm{~mm}, w=1.98 \mathrm{~mm}, d=$ $31 \mathrm{~mm}$, and the angle $\theta=90^{\circ}$. The patch antenna used here is the traditional air-filled microstrip antenna with $135 \mathrm{~mm} \times$ $122 \mathrm{~mm}$ patch size, and the proposed RFID antenna was constructed by placing a $3 \times 3$ array cross-shaped structure at the middle of the patch and ground plane as shown in Figure 1(b). The photograph of the fabricated antenna was shown in Figure 1(c).

\section{Results and Discussions}

The measurements were performed with Agilent 8753ES vector network analyzer in Airlink 3D anechoic chamber. The transmission coefficient of electromagnetic waves through the $3 \times 3$ array was shown in Figure 2. It can be seen that 


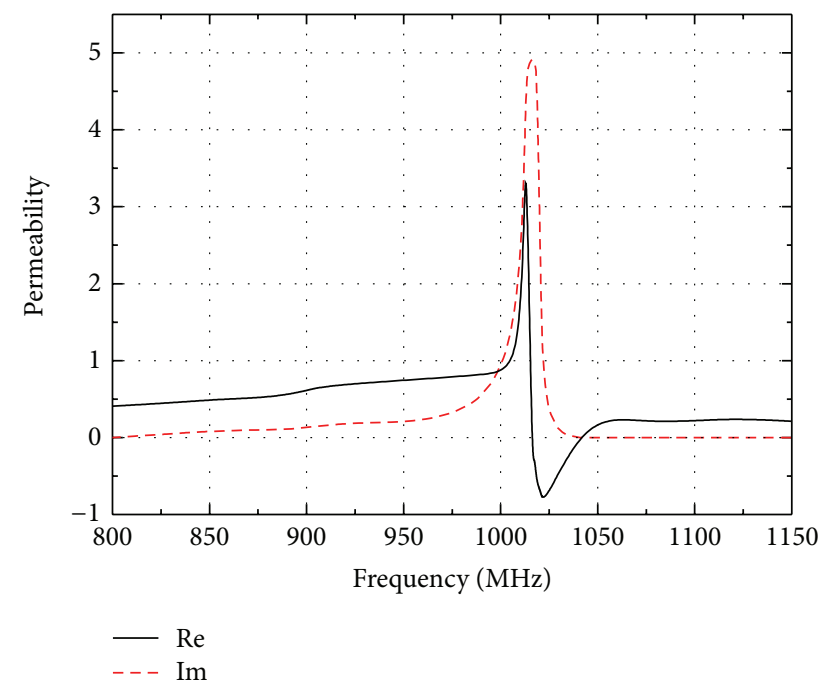

Figure 3: Permeability versus frequency for cross-shaped structure.

3 Dec 2012 20:54:52

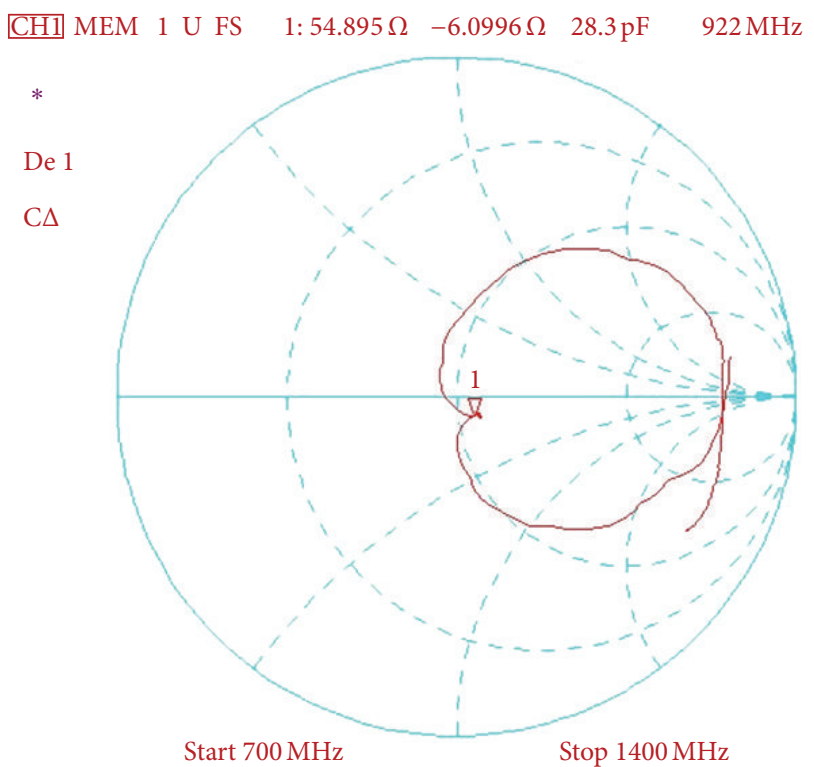

FIgURE 4: The measured Smith chart of the proposed CP RFID antenna.

the cross-shaped structure displays a dip at $1016 \mathrm{MHz}$. This phenomenon indicates that cross-shaped structure has both electric and magnetic response. Meanwhile, the permeability of this structure shown in Figure 3 was calculated through scattering parameter method. It can be seen that the permeability of the structure is negative at $1016 \mathrm{MHz}$, which implies that the designed cross-shaped fractal structure shows the behavior of LHM. Thus, it breaks through the halfwavelength restrict of traditional microstrip antenna.

The measured Smith chart of the antenna is given in Figure 4. It can be seen that the designed RFID antenna is tuned with excellent impedance matching and good circular polarization state [8]. The measured VSWR of antenna with and without the cross-shaped structure is presented

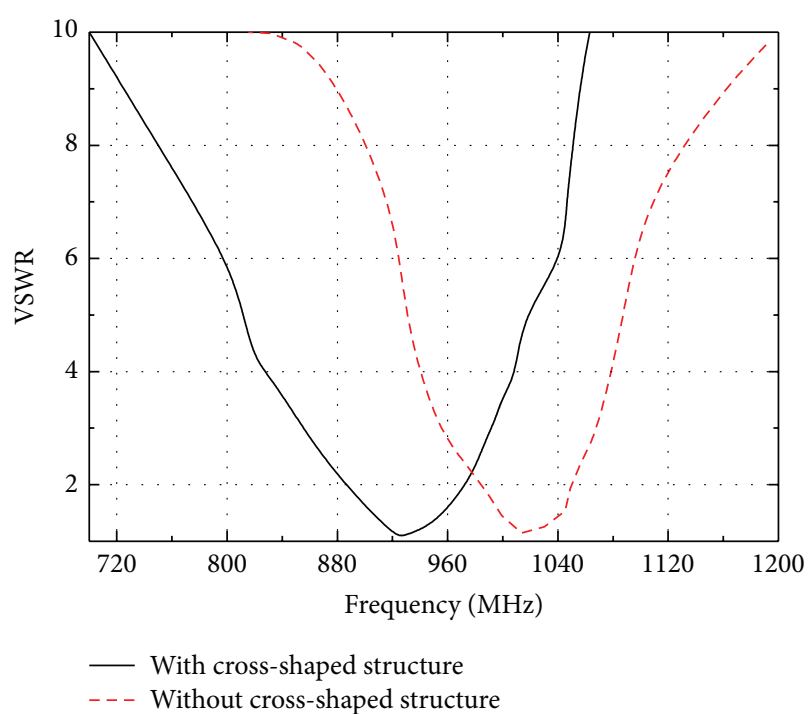

FIGURE 5: The VSWR of patch antenna with and without crossshaped structure.

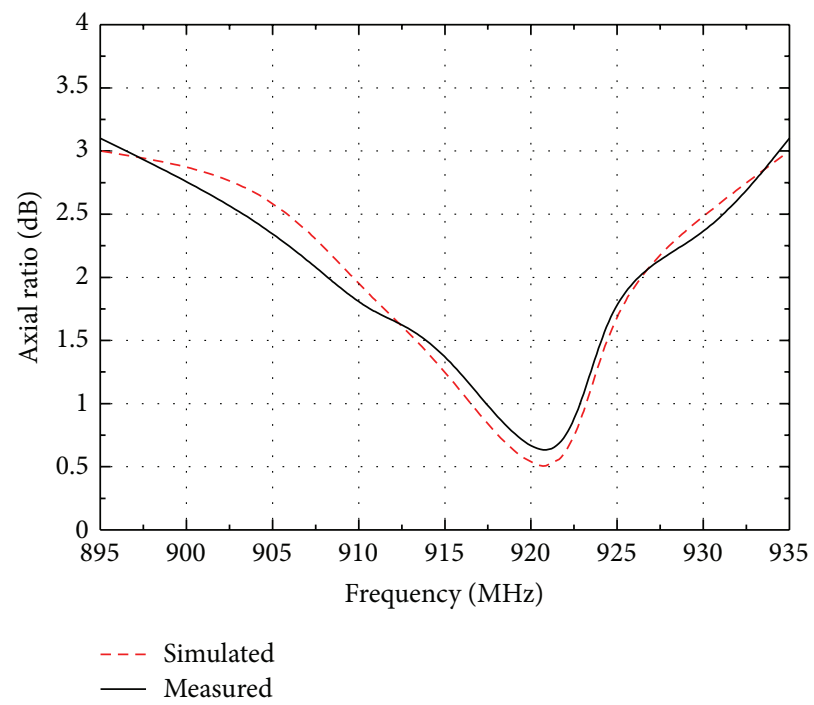

FIGURE 6: AR of the designed RFID antenna versus frequency.

in Figure 5. It is easy to see that the resonant frequency is changed from $1016 \mathrm{MHz}$ to $922 \mathrm{MHz}$ after using the designed cross-shaped LHM. Figure 6 shows the simulated and measured axial ratio (AR) of designed RFID antenna. The measured $3-\mathrm{dB}$ AR bandwidth is $4.3 \%$ for the proposed antenna and $4.5 \%$ for the traditional antenna. In addition, the simulated and measured radiation patterns for the designed antenna on the center frequency $922 \mathrm{MHz}$ are given in Figure 7. All the results for the fractal antenna are in agreement with that of the traditional patch antenna, which means that the proposed cross-shaped fractal LHM structure does not have severe influence on the radiation pattern but the resonant frequency. In other word, the proposed antenna can operate at lower frequency with the same size. 


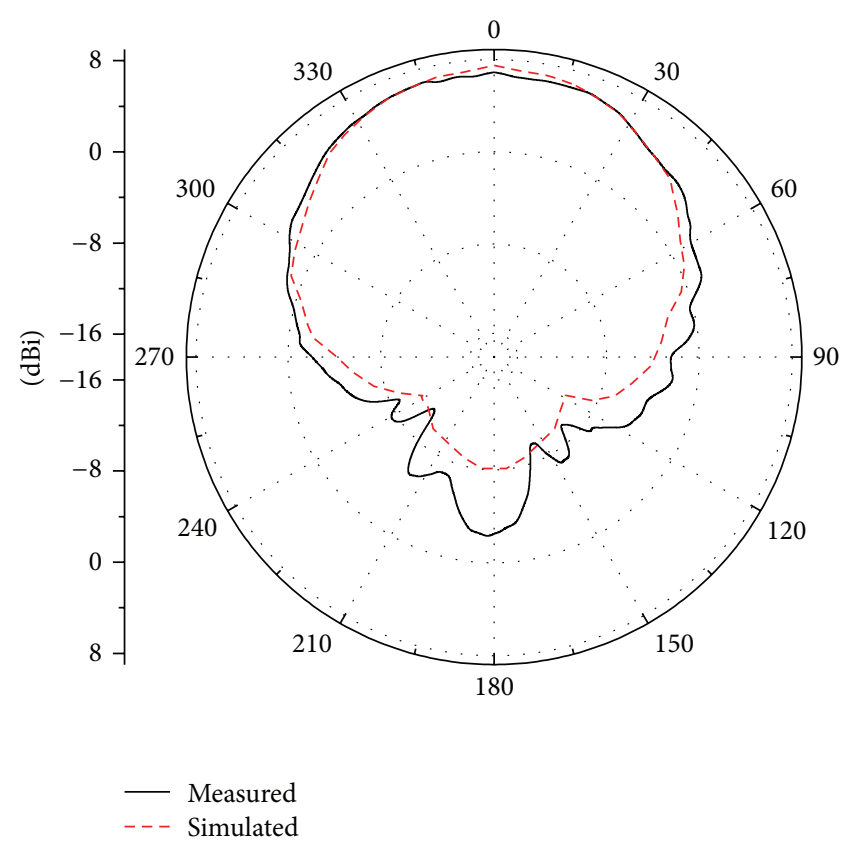

Figure 7: The simulated and measured radiation patterns at $922 \mathrm{MHz}$.

\section{Conclusion}

A novel cross-shaped fractal LHM structure has been constructed and applied to miniaturize RFID patch antenna. The measured results show that the proposed antenna has an impedance bandwidth of $8.7 \%$ at $922 \mathrm{MHz}$ with good radiation patterns. Furthermore, the proposed antenna has $10.2 \%$ size reduction compared with the traditional patch antenna.

\section{Acknowledgment}

This work is supported by the National Natural Science Foundation of China under Grant 61179021.

\section{References}

[1] V. D. Hunt, A. Puglia, and M. Puglia, RFID: A Guide to Radio Frequency Identification, John Wiley \& Sons, New York, NY, USA, 2007.

[2] J. S. Colburn, "Patch antennas on externally perforated high dielectric constant substrates," IEEE Transactions on Antennas and Propagation, vol. 47, no. 12, pp. 1785-1794, 1999.

[3] Y. L. Chow and K. L. Wan, "Miniaturizing patch antenna by adding a shorting pin near the feed probe-a folded monopole equivalent," in Proceedings of the IEEE Antennas and Propagation Society International Symposium, vol. 4, pp. 6-9, June 2002.

[4] M. H. Song and J. M. Woo, "Miniaturisation of microstrip patch antenna using perturbation of radiating slot," Electronics Letters, vol. 39, no. 5, pp. 417-419, 2003.

[5] B. B. Mandelbrot, The Fractal Geometry of Nature, W. H. Freeman, New York, NY, USA, 1983.
[6] X. Liang and C. Y. W. Michael, "A microstrip antenna with fractal multilayer substrates," Microwave Journal, vol. 43, no. 2, pp. 158-162, 2000.

[7] X. Liang and M. Y. W. Chia, "Multiband characteristics of two fractal antennas," Microwave and Optical Technology Letters, vol. 23, no. 4, pp. 242-245, 1999.

[8] G. Kumar and K. P. Ray, Broadband Microstrip Antennas, Artech House Antennas and Propagation Library. 

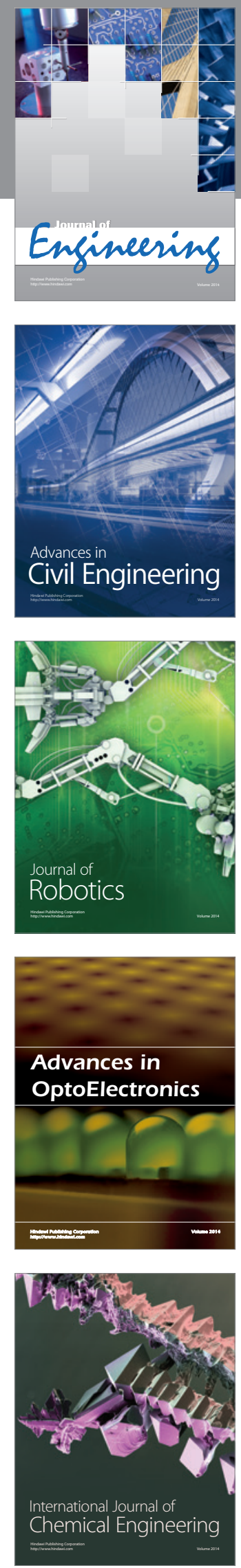

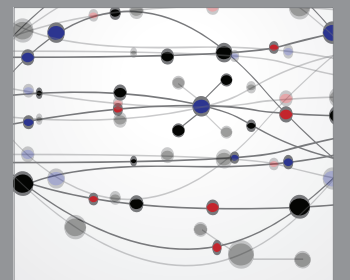

The Scientific World Journal
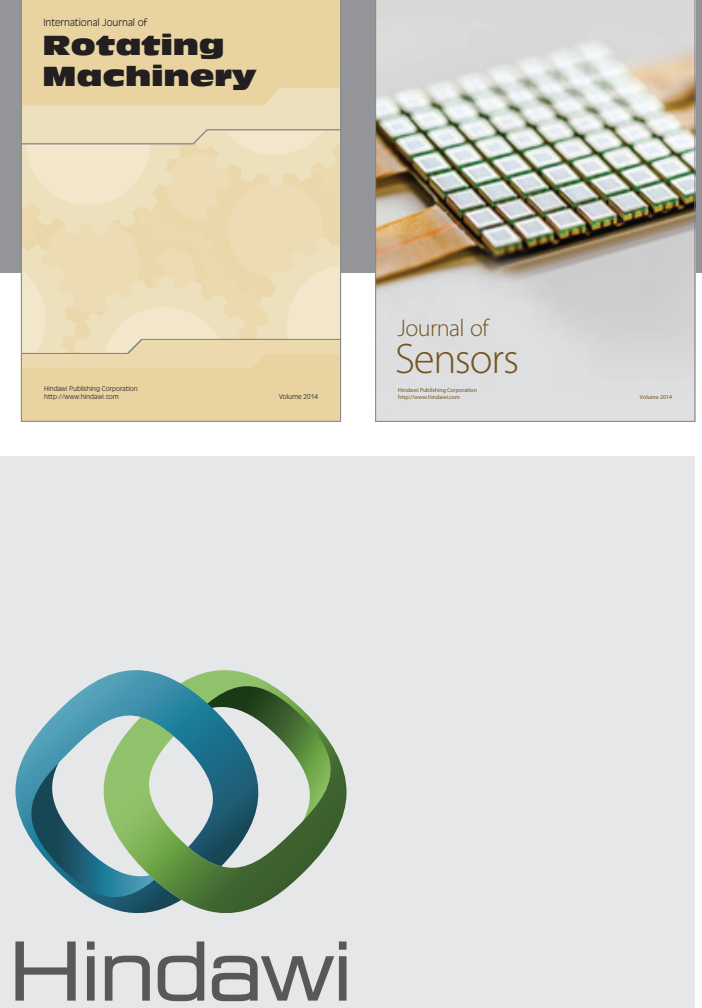

Submit your manuscripts at http://www.hindawi.com
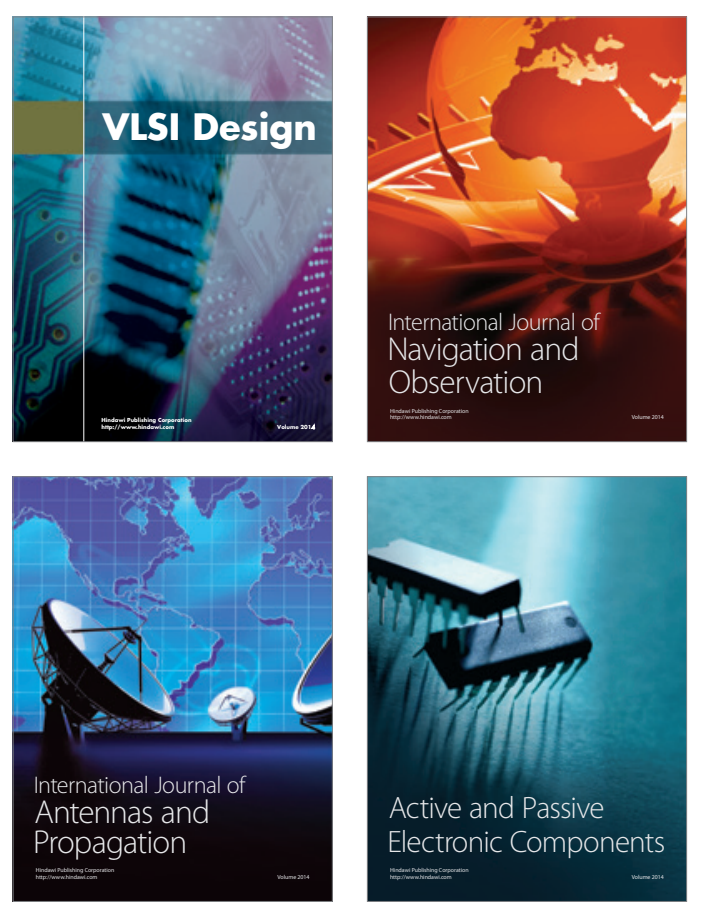
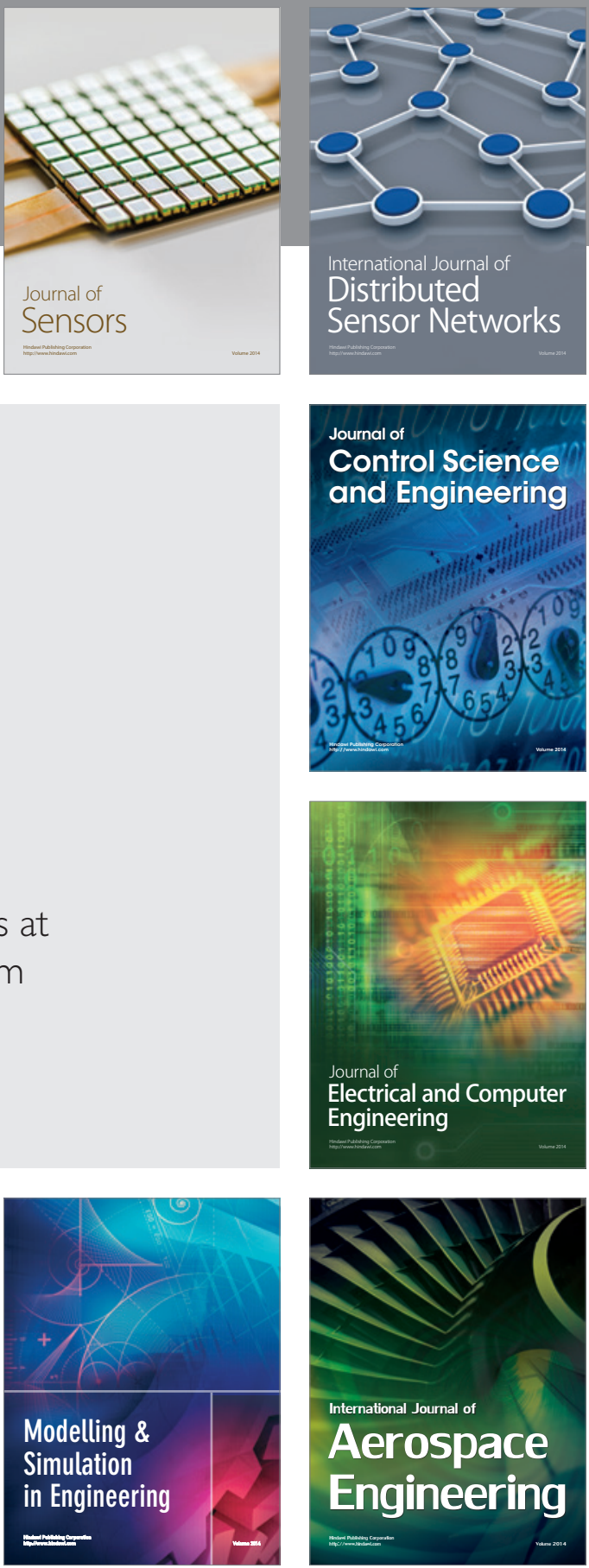

Journal of

Control Science

and Engineering
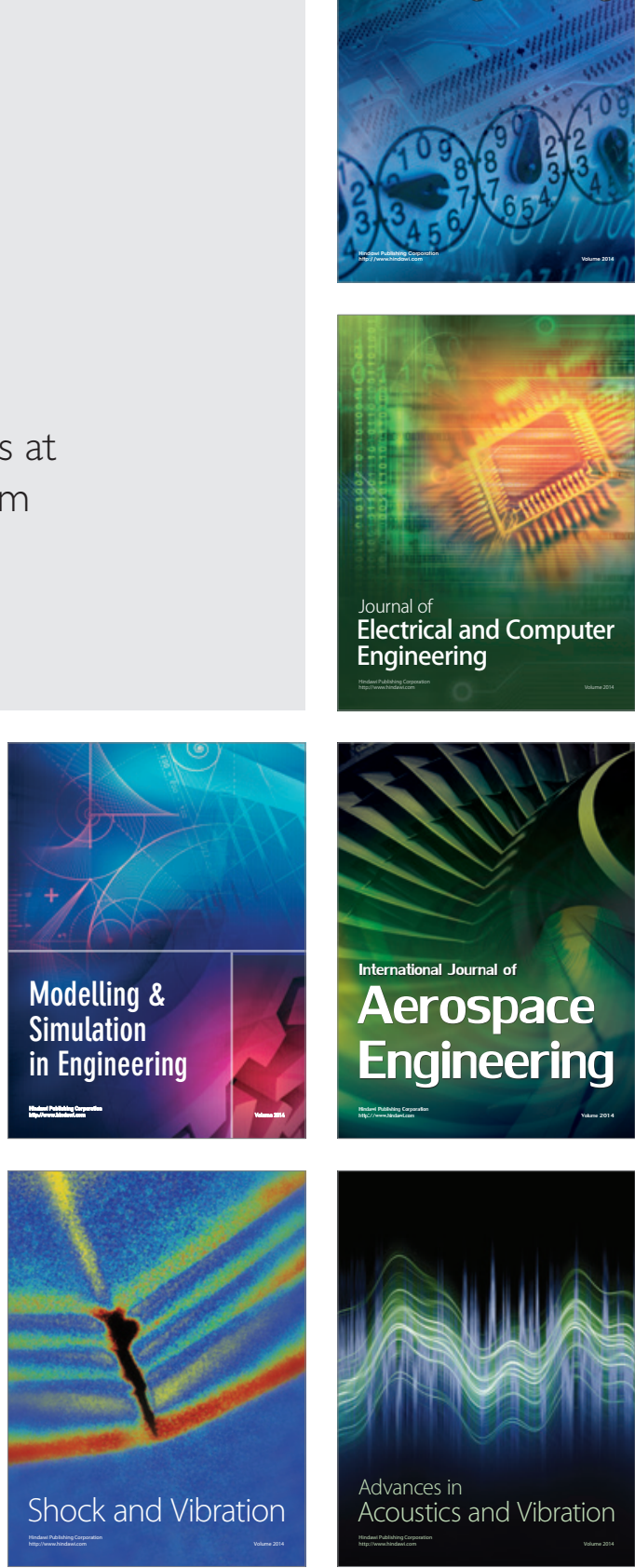